\title{
Plasma Flow and Temperature in a Gliding Reactor with Different Electrode Configurations
}

\author{
J. Sláma
}

\begin{abstract}
This paper deals with the plasma flow shape depending on the electrode form of a gliding discharge plasma-chemical reactor, and with the temperature distribution along the direction of the plasma flow in one specific electrode form. The shape of the electrodes and their mutual position has a significant influence on the design of a gliding discharge reactor and its applications. It is crucial to know the temperature distribution in the reactor's chamber design and discharge application.

Three configurations with model shapes of wire electrodes were therefore tested (low-divergent, circular, highdivergent) and the plasma flow was described. The experiments were performed in air at atmospheric pressure and at room temperature. In order to map the reactive plasma region of the flow we investigated the visible spectral lines that were emitted. The gas temperature was measured using an infrared camera.
\end{abstract}

Keywords: gliding discharge, spectra, shape of electrodes, plasma reactor, plasma flow, temperature.

\section{Introduction}

In recent years there have been many physical and technological applications of gliding discharge as "an auto-oscillating phenomenon developing between at least two electrodes that are immersed in a laminar or turbulent gas flow" [1]. The gliding discharge configuration its parameters, e.g. discharge voltage and current, injected working gas flow rate, and shape of the electrodes. In this paper we have focused on the emitted spectra and the plasma flow temperature which were observed by changing two parameters. The spectra were measured for three model shapes of electrodes (low-divergent, circular and high-divergent). Motivated by utilization of the high-divergent electrode configuration, the temperature of the gliding discharge plasma flow was measured for the high-divergent configuration and for three working gas flow rates (10, 20 and $30 \mathrm{slm})$.

\section{Experimental}

The apparatus (Figure 1) used in our experiments consisted of a plasma chemical reactor containing electrodes (copper wires $1 \mathrm{~mm}$ in diameter shaped into the required form - Figure 2), a nozzle, and the gas distribution system (flowmeter, reduction valve, piping and air compressor).

The electrodes were connected to the high voltage power source $(U=8 \mathrm{kV}, f=50 \mathrm{~Hz})$. The interelectrode distance at the point of initial discharge breakdown was $3 \mathrm{~mm}$. The reduction valve held the gas flow to average values of $Q=10,20$ and $30 \mathrm{slm}$. The experiment was performed at temperature about $21^{\circ} \mathrm{C}$, atmospheric pressure $101 \mathrm{kPa}$ and air humidity about $30 \%$.

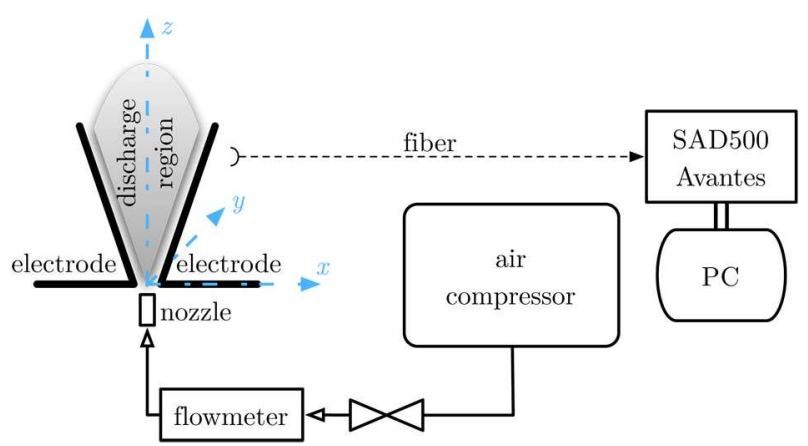

Fig. 1: Plasma chemical reactor, gas distribution system and spectroscope

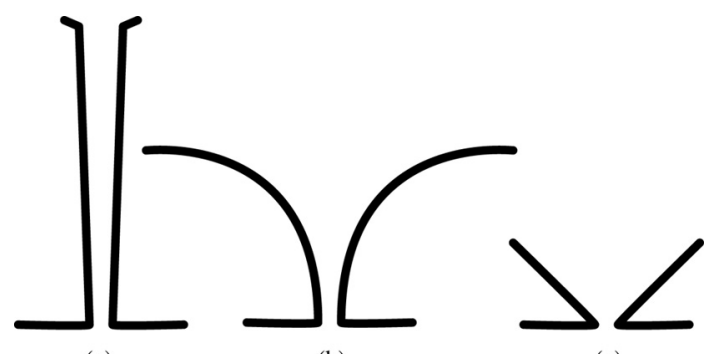

(a)

(b)

(c)

Fig. 2: Shape of the electrodes: (a) - low-divergent, (b) circular, (c) - high-divergent

\section{$2.1 \quad$ Electrodes}

Three wire electrode configurations were tested with model electrode shapes — "low-divergent", "circular" and "high-divergent" (Figure 2). Two $130 \mathrm{~mm}$ 
long straight electrodes represented the low-divergent configuration, circular electrodes were formed by a pair of ring-shaped electrodes $22 \mathrm{~mm}$ in radius, and the high-divergent configuration was two sharp triangular right angle-shaped electrodes. The model shapes of the electrodes are represented by both lowdivergent and high-divergent configurations.

The photos in Figure 3 were taken using a Nikon D90 camera and an AF-S Nikkor 18-200 mm objective. The exposure was $1 \mathrm{~s}$ shutter speed, aperture $f / 13$. ISO 200 was used for the photography of lowdivergent configuration and ISO 160 was used for the photography of circular and high-divergent configuration.

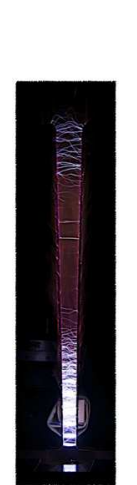

(a)

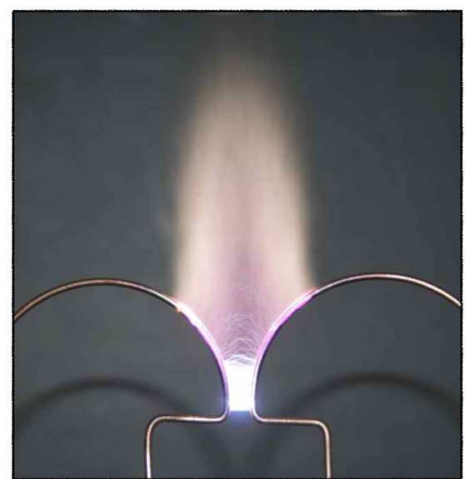

(b)

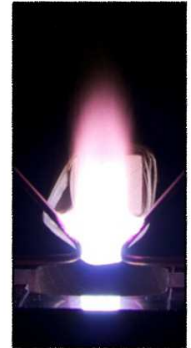

(c)
Fig. 3: Plasma flows in low-divergent (a), circular (b), high-divergent (c) electrode configuration, $Q=20 \mathrm{slm}$ (pictures not taken on the same scale)

\subsection{Temperature and visible spectra}

Temperature A basic analysis was performed of the temperatures in the gliding discharge. We used a GUIDE M8 infrared camera (Figure 4) for scanning the observed space. The resolution of the microbolometer array was $160 \times 120$ pixels with spectral range from $8 \mu \mathrm{m}$ to $14 \mu \mathrm{m}$, temperature range from $-20^{\circ} \mathrm{C}$ to $250^{\circ} \mathrm{C}$, and sensitivity $\leq 0.1 \mathrm{~K}[2]$.

For better temperature distribution visualization in the gliding discharge plasma flow we used a set of "optical fibre probes", due to the low emissivity and the low mass density of the plasma.

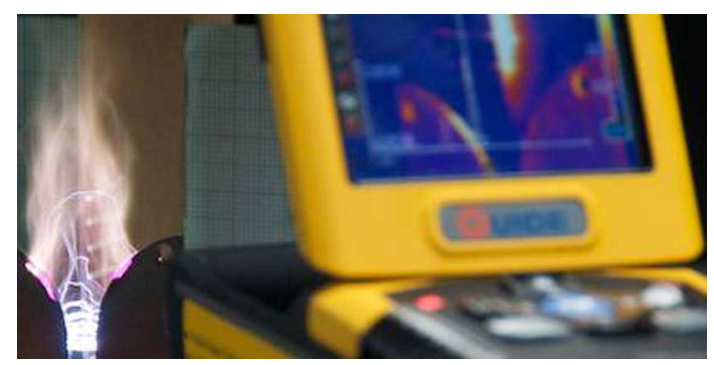

Fig. 4: GUIDE M8 infrared camera for measuring thermal emission

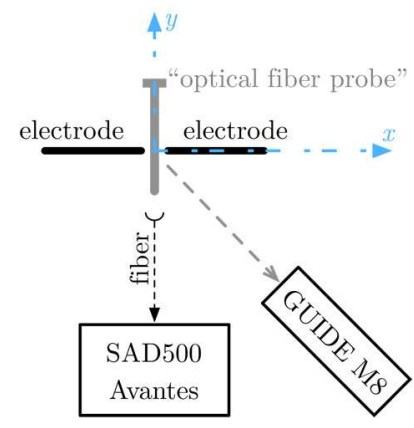

Fig. 5: Horizontal projection of the experimental setup with an SAD500 Avantes spectrometer and GUIDE M8 infrared camera (see also Figure 1)

The optical fibre probes were made of $50 \mu \mathrm{m}$ fibre (taken from the GUMT206 Fibre Optic Cable (8 fibre $50 / 125 \mu \mathrm{m})[3])$. The probes were placed in a straight line in the $x y$-plane (Figure 5 and 1 ) with a mutual distance of $5 \mathrm{~mm}$ in the direction of the positive $z$-axis direction - all probes were perpendicular to the plasma flow and were heated by circumfluent plasma. The GUIDE M8 infrared camera was placed as shown in Figure 5. The optical fibre probes were scanned by infrared camera approx. at an angle of $45^{\circ}$. The distance from the camera to the probes was about $270 \mathrm{~mm}$.

Spectra A SAD500 Avantes spectrometer with fiber for transmission of the light from the discharge was used for measuring of the visible spectra. The spectral range of the spectrometer was from $190 \mathrm{~nm}$ to $861 \mathrm{~nm}$. The resolution of the spectrometer was $0.328 \mathrm{~mm}$. The spectrometer fiber was set perpendicular to the $z$-axis in the direction of the $y$-axis (Figure 5). To scan the whole plasma flow axial area, the fiber was moved axially in the $z$-axis direction.

\section{Interpretation}

The spectra in the visible wavelength range were taken in all three configurations. As the plasma region was relatively small, the temperature was measured indirectly along the $z$-axis only in the highdivergent electrode configuration.

\subsection{Spectra}

The ionized flow regions were registered by visible spectral line intensities. Each intensity value in the graphs in Figures 6, 7 and 8 represents the average value of 7 measurements.

Based on the NIST database [4], we found four spectral lines $-\lambda_{\mathrm{A}}, \lambda_{\mathrm{B}}, \lambda_{\mathrm{C}}$ and $\lambda_{\mathrm{D}}-$ typical for low temperature plasma in air.

$$
\begin{aligned}
& \lambda_{\mathrm{A}}=463.061 \mathrm{~nm} \text { NIII } 2 \mathrm{~s} 2 \mathrm{p}\left({ }^{3} \mathrm{P}^{\circ}\right) 4 \mathrm{p}-2 \mathrm{~s} 2 \mathrm{p}\left({ }^{3} \mathrm{P}^{\circ}\right) 5 \mathrm{~s}, \\
& \lambda_{\mathrm{B}}=463.885 \mathrm{~nm} \text { OII } 2 \mathrm{~s}^{2} 2 \mathrm{p}^{2}\left({ }^{3} \mathrm{P}\right) 3 \mathrm{~s}-2 \mathrm{~s}^{2} 2 \mathrm{p}^{2}\left({ }^{3} \mathrm{P}\right) 3 \mathrm{p}, \\
& \lambda_{\mathrm{C}}=500.113 \mathrm{~nm} \mathrm{NII} ; 2 \mathrm{~s}^{2} 2 \mathrm{p}\left({ }^{2} \mathrm{P}^{\circ}\right) 3 \mathrm{p}-2 \mathrm{~s}^{2} 2 \mathrm{p}\left({ }^{2} \mathrm{P}^{\circ}\right) 3 \mathrm{~d}, \\
& \lambda_{\mathrm{D}}=567.602 \mathrm{~nm} \mathrm{NII} ; 2 \mathrm{~s}^{2} 2 \mathrm{p}\left({ }^{2} \mathrm{P}^{\circ}\right) 3 \mathrm{~s}-2 \mathrm{~s}^{2} 2 \mathrm{p}\left({ }^{2} \mathrm{P}^{\circ}\right) 3 \mathrm{p} .
\end{aligned}
$$




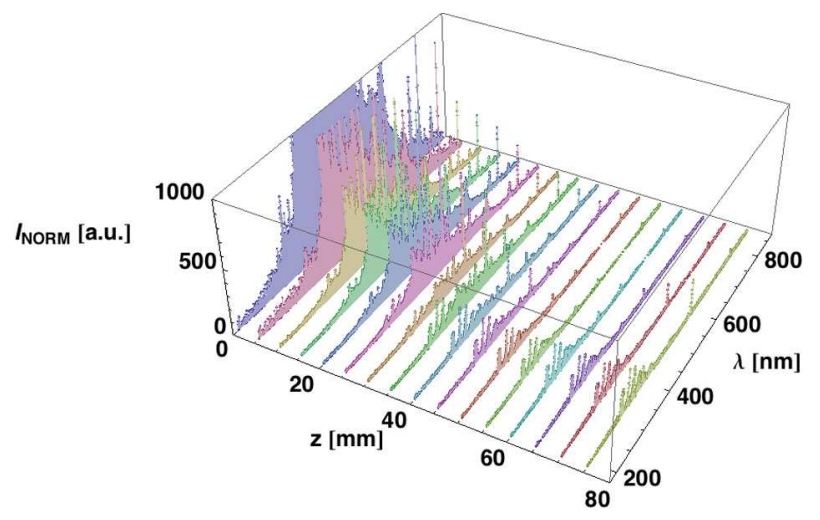

Fig. 6: Intensity distribution along the $z$-axis in the lowdivergent electrode configuration, $Q=20 \mathrm{slm}$

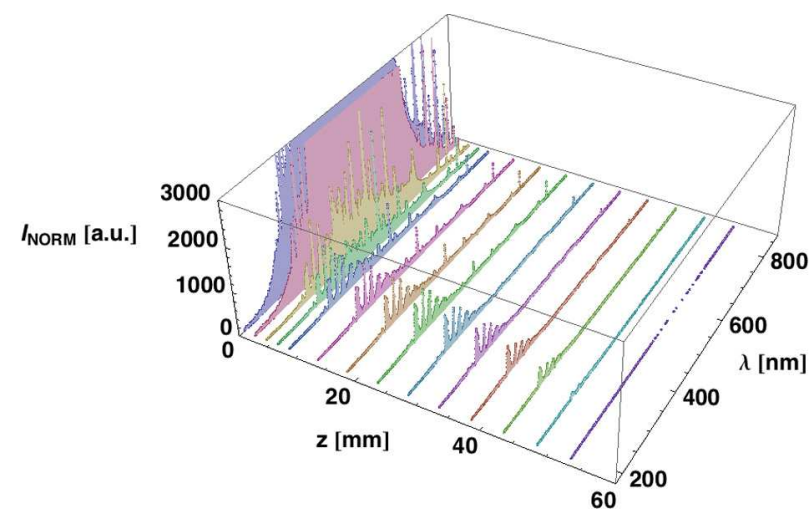

Fig. 7: Intensity distribution along the $z$-axis in circular electrode configuration, $Q=20 \mathrm{slm}$

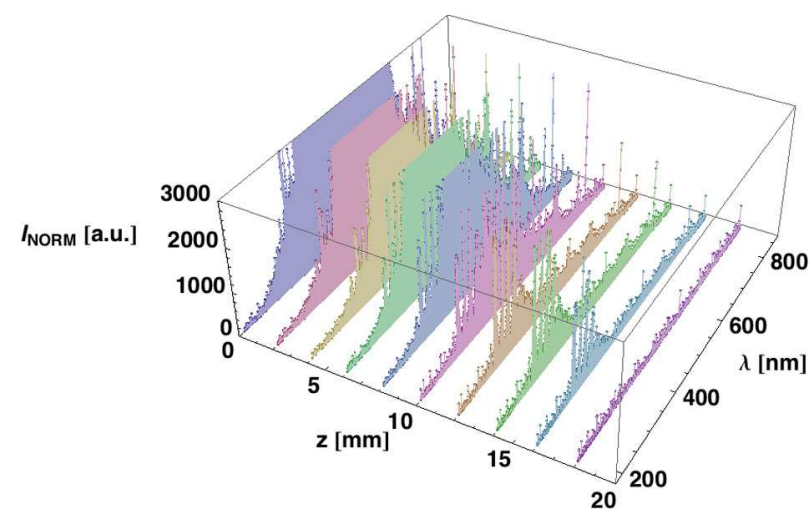

Fig. 8: Intensity distribution along the $z$-axis in highdivergent electrode configuration, $Q=20 \mathrm{slm}$

In the UV spectra range we also found the first negative system of $\mathrm{N}_{2}^{+}$and the second positive system of $\mathrm{N}_{2}$ in all three measured spectra. This is shown in Figures 6,7 and 8 as the visible band around wavelength $\lambda_{\mathrm{E}} \sim 390 \mathrm{~nm}$.

To detect the plasma particles excitations it is possible to observe the measured spectra, e.g. the peaks $\lambda_{\mathrm{A}} \ldots \lambda_{\mathrm{D}}$ and the spectral band $\lambda_{\mathrm{E}}$. The intensity distribution of peaks $\left(\lambda_{\mathrm{A}} \ldots \lambda_{\mathrm{E}}\right)$ in the $z$-axis direction (Figure 9) corresponds to the shape of the plasma region (Figure 3(c)). From the results, we expect that the most excited plasma region will be found in the ignition area (Figures 6, 7, 8 and 9). The plasma distribution for the individual configurations in the interelectrode region can be described as follows:

- In the low-divergent configuration (Figures 3(a) and 6), a typical band $\lambda_{\mathrm{E}} \sim 390 \mathrm{~nm}$ was found along the $z$-axis through the whole interelectrode region (Figure 6) and plasma channels were originated on the whole length of the electrodes.

- In the circular configuration, the plasma region was twice as long as the radius of the circular electrodes (Figures 3(b) and 7). An outstanding " $\lambda_{\mathrm{E}}$ band" was also observed.

- In the high-divergent configuration the plasma outreached the interelectrode region by about $10 \mathrm{~mm}$ (Figures 3(c), 8 and 9). A sharp transition was found between the ionized and neutral parts of the flow $(z \sim 20 \mathrm{~mm}$ on Figure 9$)$.

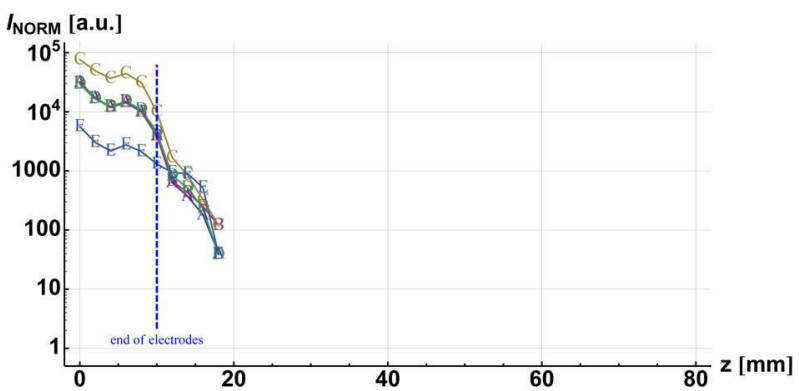

Fig. 9: Intensity distribution along the $z$-axis in highdivergent electrode configuration, $Q=20 \mathrm{slm}$

\section{$3.2 \quad$ Temperature}

Motivated by actual utilization of the high-divergent electrode configuration, we measured the temperature distribution in the interelectrode region along the $z$-axis in this configuration. To visualize the temperature in the infrared camera more easily we scanned the interelectrode space with an added set of optical fiber probes (Figure 5). From the overall infrared picture (Figure 10) we selected the required cross-section (green line) which included the scanned "probes" heated by the gas flow.

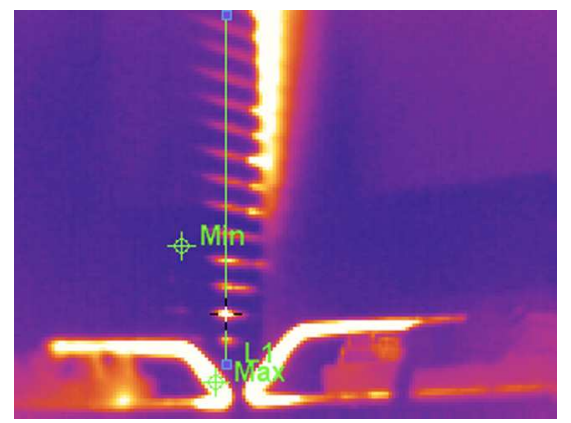

Fig. 10: Infrared picture from the GUIDE M8 infrared camera 
Five infrared pictures were taken (Figure 10) by the GUIDE M8 for each gas flow $Q=10,20$ and $30 \mathrm{slm}$. Then the temperatures of the probes were extracted and the distribution containing the mean values was plotted for the gas flows (Figure 11).

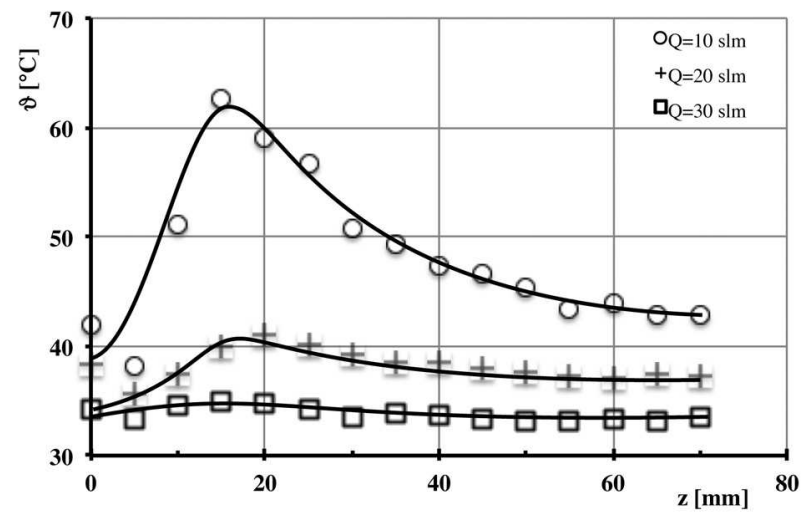

Fig. 11: Temperature distribution along the $z$-axis for gas flows $Q=10,20$ and 30 slm (high-divergent configuration)

As shown in the graph (Figure 11), the gliding discharge axial temperature distribution depended on the gas flow. With increasing gas flow values $Q$, the temperature of the probe decreased. The maxima of the curves in Figure 11 were approximately at the end of the discharge region, e.g. $z \sim 20 \mathrm{~mm}$.

\section{Summary}

The connection between three basic electrode profiles (low-divergent, circular, high-divergent) and the appropriate plasma flow shapes in the gliding discharge reactor has been studied. The experiments were carried out in the air at room temperature and atmospheric pressure. The most ionized region was identified by the visible spectral lines of the observed plasma, and was located in the ignition area.

In the high-divergent configuration, the temperature was measured indirectly along the perpendicular axis for three gas flow values. The hottest region was found at the end of the plasma region, and its temperature depended on the gas flow, i. e. with increased flow rate the overall temperature dropped.

Our results can be helpful for finding suitable gliding reactor electrode shapes, i. e. the shapes of the plasma flow in the reactor, and in technological applications of the discharge (e.g. decompositions [5], surface treatment, etc.).

\section{Acknowledgement}

Research described in the paper was supported by the Czech Technical University in Prague grant No. SGS10/266/OHK3/3T/13 and by the SurfaceTreat, a.s., Czech Republic.

\section{References}

[1] Bo, Y. et al: The Dependence of Gliding Arc Gas Discharge Characteristics on Reactor Geometrical Configuration. Plasma Chem Plasma Process (2007) 27:691700, Springer Science+Business Media, LLC 2007.

[2] Reseller webpage, online: March $10^{\text {th }} 2011$, http://www.guide-infrared.cz/termokamery-rucni/ termokamera-guide-m8.

[3] Datasheet, online: March $10^{\text {th }} 2011$, http://www.anixter.com/axecom/AXEDocLib.nsf/ (UnID)/DE885588DEFC8CE1862575A200611206/ \$file/Fibre.pdf.

[4] NIST: Atomic Spectra Database Lines Form, online: $1^{\text {st }}$ April 2010, http://physics.nist.gov/PhysRefData/ASD/ lines_form.html.

[5] Krawczyk, K., Ulejczyk, B.: Decomposition of Chloromethanes in Gliding Discharges. Plasma Chemistry and Plasma Processing, vol. 23, no. 2, June 2003, p. 265-281.

\section{About the author}

Jan Sláma was born in Jablonec nad Nisou in 1982. He was awarded his bachelor degree in 2007 and his master degree in 2010 from FEE CTU in Prague. He started his PhD studies on plasma physics at the Department of Physics, FEE CTU in Prague in 2010. He designed a plasma-chemical reactor for ADBD applications for his bachelor project, and implemented a high voltage middle frequency power source for $\mathrm{ADBD}$ reactors for his master project. He is currently working on characterizing gliding discharge and applying it in various technical fields.

Jan Sláma

E-mail: slamajan@fel.cvut.cz

Department of Physics

Faculty of Electrical Engineering

Czech Technical University in Prague

Technická 2, 16627 Praha, Czech Republic 